\title{
Broadcast Services and Topology Control in Ad-Hoc Networks
}

\author{
Flaminio Borgonovo, Matteo Cesana, Luigi Fratta \\ DEI, Politecnico di Milano \\ borgonov, cesana, fratta@elet.polimi.it
}

\begin{abstract}
The design of effective Medium Access Control protocol for ad hoc network is a highly challenging task due to the characteristics of such networks. The medium is shared and unreliable by definition, thus the access mechanism should be robust to channel errors and collisions. Furthermore, all the coordination functions must be totally distributed in a pure ad hoc environment.

In this paper we address the issue of designing an effective MAC protocol for Inter Vehicular Communications (IVC) networks with a particular focus on broadcast services and topology control capabilities.
\end{abstract}

\section{Introduction}

The design of ad hoc networks has recently attracted a lot of attention, mainly because many characteristics of such networks, especially in a highly mobile environment, make the design of a prompt, efficient, flexible, and reliable MAC very difficult.

Applications of mobile ad hoc networks can range from military ones, where networks need to be deployed immediately without the support of either base stations or fixed network infrastructures, to inter-vehicle communications, designed for both traffic safety enhancement and entertainment purposes. The inter-vehicle communications application poses the most stringent requirements, due to a highly variable topology and to the need to provide a continuous exchange of broadcast information to support traffic control applications [1].

Within this application field, to achieve a reduction of 50\% in road accidents within 2010 [2], the European Commission has promoted an integrated safety program, including: traffic management, driver assistance, passive safety, and emergency management. To this end, a special interest exists in developing Intelligent Transportation System (ITS) services based on wireless vehicular communications both among vehicles (Inter Vehicles Communication, IVC) and between vehicles and fixed infrastructure at the side of the road (Vehicles to Road Communication, VRC) [3] [4].

Typical applications of IVC and VRC include:

- Information and Warning Functions (IWF): this service requires to transmit alarm information regarding stopped vehicles, traffic accidents, traffic intensity and congestion, road surface conditions. Such an information should be promptly available at the designated receivers and therefore requires the transmission of short aperiodic broadcast packets with high priority and stringent delay requirements.

- CoOperative Driver Assistance (CODA): this application is helpful for handling and managing merging situation, overtaking and lane changing manoeuvres. A direct addressing of neighboring vehicles and a reliable unicast communication link with low latency are 
needed. Broadcast functionality might be useful for the CODA functions as well, e.g. to send a gap request to all nearby vehicles.

Most of the applications in IVC networks, as those mentioned above, require the vehicles to continuously "broadcast" some background information, such as cruise parameters [5]. This information is intrinsically broadcast because directed mostly to neighboring vehicles. In such a scenario, the MAC level should be able to provide the upper layers with prompt and reliable broadcast channels.

Almost none of the standardized wireless technologies, like the IEEE802.11, seems to be well suited for IVC applications, since the broadcast service they implement is highly unreliable.

Furthermore, due to the variable environment, in mobile ad hoc networks all protocols and coordinating functions must be totally distributed. Since the topology of the network has a dramatic impact on its performance, an effective topology control algorithm should be integrated at MAC level in order to guarantee good performance in terms of throughput and network connectivity with dynamic and variable network topologies. These kinds of algorithms often operate in a fully distributed way by modulating the transmitting power of each terminal according to the network situation it is experiencing.

In this paper we address the issue of designing an effective MAC protocol for IVC networks with a particular focus on broadcast services and topology control capabilities. In Section 2 we briefly discuss the effectiveness of IEEE 802.11 as far as the broadcast service is concerned. Further on, we highlight the issue of designing an effective broadcast support at MAC level by reviewing some of the existing research results. Section 3 comments the need of implementing a topology control in an ad hoc network environment. In Section 4 we briefly describe the features of the ADHOC MAC proposed in [6,7], to support a reliable broadcast service and we present a novel and effective topology control algorithm which can be easily integrated within ADHOC MAC. Section 5 presents numerical results on th performance of broadcast and topology control algorithm. In Section 6 some concluding remarks are given.

\section{MAC Design and Broadcast services for Ad Hoc Networks}

Since the wireless medium is inherently a shared resource, the effectiveness of the mechanism that controls the access to the channel determines the capacity of the network and has a dramatic impact on system complexity and cost.

The ad hoc networks architecture is completely distributed, that is, no logical distinction among the entities composing the network exists. No central stations, like in cellular networks, can control and rule the channel access, nor coordinate the operations of the end users. Everybody in a pure ad hoc network is contemporary base station and end user.

MAC protocols for ad hoc networks must cope with some problems typical of a wireless environment:

- Unreliable Links: wireless media is unreliable since any transmission can suffer a high bit error rate, due to multi-path, fading and shadowing phenomena. Furthermore, wireless nodes can move around at non negligible speed.

- Collision: wireless media is shared, so some kind of access control should be implemented at MAC level in a distributed way.

- Power Consumption: power saving issues should be further taken into account in MAC design since wireless nodes are often battery powered.

The existing MAC protocols for ad hoc networks can be classified in two categories: contention free and contention based. The contention free protocols avoid collisions during access 
phase through a radio resource scheduling algorithm which assigns each users its own transmission slot on a time division basis [8] [9], thus they are based on some kind of Time Division Multiple Access (TDMA) protocol. These solutions work with a synchronous communication scenario where the radio channel is divided into several time slots, and each wireless station has knowledge of this assignment. This solution reduces bandwidth wastage due to collision but it needs a complex slot scheduling algorithm, which has to be totally distributed.

On the other hand, the basic principle of contention based protocols is competition, i.e., wireless nodes compete to gain the access to the channel, and possible collisions are resolved by randomized retransmission procedures. These protocols are based on ALOHA and on CSMA $[10,11]$. Strength points of these protocols are simplicity and good performance in low traffic situations. Unfortunately, these solutions doesn't scale well at high loads since collisions tend to become systematic and affect the network performance. For this reason, mechanism of collision avoidance are often adopted in contention based access protocols.

The Distributed Coordination Function (DCF) mechanism adopted in the IEEE802.11 standard is the most used contention based MAC protocol [12]. It implements some concepts proposed in MACA (Multiple Access with Collision Avoidance) protocol [13] and in MACAW (Multiple Access with Collision Avoidance for Wireless networks) protocol [14]. In details, after a physical carrier sensing phase, a handshaking phase can be used in order to alleviate hidden nodes interference and minimize the number of exposed nodes. A station willing to transmit sends out a Request To Send (RTS) to probe the availability of the destination. The destination, if available, answers with a Clear To Send (CTS) packet. All the stations overhearing one of the two control packets are prevented from accessing the channel. The RTS/CTS exchange is preceded with a physical carrier sensing phase, in order to limit collisions among control packets.

As far as the broadcast service is concemed, two concepts of broadcasting can be defined: single-hop broadcast and multi-hop broadcast. The former lets a packet to be correctly received by any of the recipients within the transmitting range of the transmitter. All the wireless transmissions can be seen as a single hop broadcast, provided that no collision can happen at one or more of the designated recipients. Due to radio range limitations, single-hop broadcasting does not cover all terminals of the network. The multi-hop broadcast, requires a mechanism that enables a packet to be reached by all the nodes in the network. Relaying and forwarding functions must be performed by "intermediate" terminals.

In IEEE 802.11 MAC protocol, no RTS/CTS exchange is used for the transmission of broadcast frames, regardless of their length. Further, no acknowledgement (ACK) is transmitted by any of the recipients of the broadcast frame. Therefore, the single hop broadcast service implemented in IEEE802.11 is highly unreliable since the sender cannot detect possible collisions [12]. Many efforts have been done in order to enhance the reliability of the IEEE802.11 single hop broadcast service [15] [17] [18], but no definite clear solution has been proposed.

Most of the advanced multi hop broadcast protocols, such as the tree-based protocol in [19], do not work well for ad hoc networks due to the dynamic nature of the network topology. Hence, the flooding approach and its variants, have been proposed as the preferred means for multi-hop broadcast service [20]. In flooding, each station retransmits a broadcast packet just once until all terminals are reached. Such a procedure is highly inefficient in networks that present an high degree of connectivity since it generates many redundant retransmissions. In addition this procedure suffers from the broadcast storm problem if integrated with random access procedures [21], since neighbor nodes are likely to re-transmit a broadcast packet almost at the same time, causing massive collisions.

As already pointed out in the previous section, the above drawback is very critical in vehicular control applications, where vehicles continuously "broadcast" some background information, such as cruise parameters [5]. This information, furthermore, is intrinsically single-hop broadcast because directed mostly to neighbor vehicles, and a flooding procedure would saturate the whole network with useless information [20]. 


\section{Topology Control in Ad Hoc Networks}

Power control procedures that adapt the terminals transmitted power according to network needs are widely and effectively used in wireless systems.

For instance, cellular systems often resorts to centralized power control procedure to provide a satisfactory Signal to Noise Ratio (SIR) to all the connections and more generally to enhance the system performance.

On the other hand, in ad hoc networks the motivations for adopting power control procedures are even stronger and manifold.

Firstly, power control schemes are adopted for power and energy saving reasons, which are a must because of the limited amount of energy available at the battery-powered wireless terminals [22] [23] [24].

Secondly, power control procedures can be used to control the topology of the network with the purpose of improving the shared resource reuse [25], limiting the collisions among concurring transmissions [26] and providing guaranteed connectivity [27].

Topology control capabilities are of utmost importance in ad hoc applications such as IVC, where the mobile terminals rapidly move. Think of the situation of a car driving from a low populated countryside area into an urban densely populated one. The TX/RX device transmission range, that must be large in the countryside to provide connectivity, needs to be drastically reduced in the urban area to limit the number of neighboring terminals. This can be achieved by controlling the transmitted power which should be set in order to optimize the trade off between network capacity and connectivity. The higher is the transmitted power the higher is the network connectivity and the smaller is the network capacity due to the reduced radio resource reuse.

The basics of many of the proposed algorithm is to control the number of each terminal's neighbors in a distributed way $[28,29,30,31]$. Namely, each terminal gathers information on the number of neighboring stations and adapts the level of transmitted power to maintain almost constant this number. The algorithms available in the literature mainly differ on the methods to get the information of the neighboring terminals and on the steps of the power update procedure.

In the next section we present a topology control algorithm which can be easily implemented with the information at layer 2 provided by ADHOC MAC briefly reviewed in the next section.

\section{The ADHOC-MAC protocol}

ADHOC MAC operates with a time slotted structure, where slots are grouped into virtual frames (VF) of length $\mathrm{N}$, and no frame alignment is needed. The slotting information can be explicitly provided by external sources, such as GPS, or implemented in a distributed way. In the following, we assume an environment where the terminals can be grouped into clusters such that all the terminals of a cluster are interconnected by broadcast radio communication. Such a cluster is defined as One-Hop $(\mathrm{OH})$. A terminal can belong to more than one $\mathrm{OH}$-cluster, leading to the case of non disjoint clusters. The union of $\mathrm{OH}$-clusters with a common subset is called a Two-Hop (TH) cluster. An example of such an environment is shown in Figure 1 where seven terminals are grouped into three $\mathrm{OH}$-clusters forming one $\mathrm{TH}$-cluster.

\subsection{RR-ALOHA}

For a correct operation, the ADHOC MAC needs that each active terminal has assigned a Basic CHannel $(\mathrm{BCH})$, corresponding to a slot in the VF, which is a reliable single hop broadcast channel not suffering from the hidden-terminal problem. This is obtained in a distributed way by the RR-ALOHA protocol, where, as in R-ALOHA, contention is used to get access to an available slot in the frame and, upon success, the same slot is reserved in the following frames and no longer accessed by other terminals until it is released. Since the ad hoc environment is 


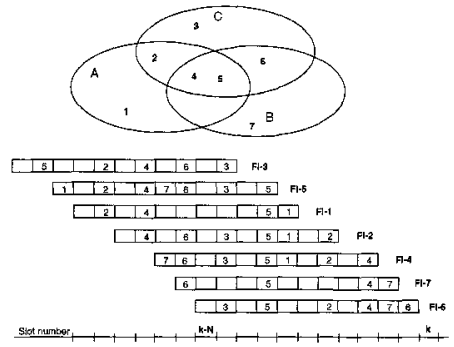

Figure 1: Example of the FI information propagated by the terminals 1.7 in the one-hop clusters $A, B$, and $C$ represented by ellipses.

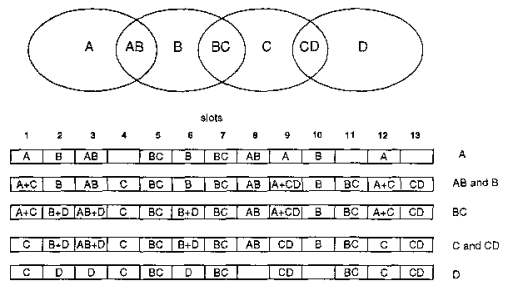

Figure 2: An Example of how terminals belonging to the labelled disjoint areas perceive reservation of slots.

not fully broadcast, the information needed for the RR-ALOHA correct operation is provided to all terminals by means of the BCHs.

Each transmission on the $\mathbf{B C H}$ contains, besides the data, a control field named Frame Information (FI). The FI is a vector with $\mathrm{N}$ entries specifying the status of each of the $\mathrm{N}$ slots preceding its transmission, as observed by the transmitting terminal itself. The slot status can be either BUSY or FREE: it is BUSY if a packet has been correctly received or transmitted by the terminal, otherwise it is FREE. In the case of a BUSY slot the FI also contains the identity of the transmitting terminal.

Based on received FIs, each terminal marks a slot, say slot $k$, either as RESERVED, if slot $\mathrm{k}-\mathrm{N}$ is coded as BUSY in at least one of the FIs received in the slots from $\mathrm{k}-\mathrm{N}$ to $\mathrm{k}-\mathrm{l}$, or as AVAILABLE, otherwise.

As in R-ALOHA an available slot can be used for new access attempts. Upon accessing an AVAILBLE slot, terminal $\mathrm{j}$ will recognize after $\mathrm{N}$ slots (a time frame) its transmission either successful, if the slot is coded as "BUSY by terminal $\mathrm{j}$ " in all the received FIs, or failed, otherwise.

The correctness of the above procedure in setting up a channel has been proved in [6] for a general network configuration, showing that no attempt at access can be made in a slot already in use when such access would cause a collision.

For the sake of simplicity, we just consider here the single TH-Cluster network of Figure 1. According to the RR-ALOHA procedure we can state the following. All terminals belonging to the same TH-cluster mark the slots in the same way. In fact, all the terminals receive the FI generated by the terminals belonging to the TH-cluster common set (terminals 4 and 5 in Figure 1) and such FI concerns all the transmissions in the TH cluster itself. A single BUSY code is enough to force a RESERVED slot. Similarly, any slot signaled as BUSY is recognized as RESERVED by all the terminals in the TH cluster and therefore, since a RESERVED slot cannot be accessed, no other terminal within the TH-cluster can transmit, and no collision will occur.

It is worth noticing that the transmission of terminals belonging to disjoint $\mathrm{OH}$-clusters cannot collide, and the slots can be reused. However, since OH-Clusters can overlap, the slot reuse in an $\mathrm{OH}$-cluster can still be constrained if the same slot is in use in the non disjoint $\mathrm{OH}$ cluster.

To illustrate this effect Figure 2 shows an example of the slot marking, observed at different terminals, where the area labels also denote terminals in the area. For the sake of clarity the RESERVED slots are represented by the corresponding terminal identity. Note that some terminals can receive FIs denoting the same BUSY slot for different terminals. This is the case of terminal B: it receives FI from AB, denoting slot 1 BUSY to terminal A, but also receives 
FI from terminal BC, denoting slot 1 BUSY to terminal $\mathrm{C}$ (the reservation is marked as $\mathrm{A}+\mathrm{C}$ ). In fact, quite correctly, the two terminals in $\mathrm{A}$ and $\mathrm{C}$ can transmit in slot 1 because $\mathrm{A}$ does not detect the FI from $B C$ and $C$ does not detect the FI from AB. Similarly, terminals lying at least three hops apart can reuse the same slot as they can never collide with each other. This shows that the goal of avoiding collisions is achieved with an optimal use of slots.

Thus it can be seen that the procedure achieves the proposed goal of setting up channels with no interference if the cluster configuration does not change.

However, when the clusters merge because of terminal migration or activation, transmission collision in established channels can still occur. In fact, transmissions in a given slot, properly reserved for different terminals belonging to disjoint clusters would, upon merging, collide at the new common terminals. The RR-ALOHA procedure enables the colliding terminals to become aware of any collision. When a collision is discovered, the slot is released and a new set up procedure is started. The frequency of this situation depends on the variations of the network topology due to the activation of new terminals and the mobility of the active ones.

The BCH gained by R-ALOHA can also be used to assure additional MAC services such as a fast and reliable single hop broadcast channel, and a signaling channel to dynamically reserve additional channels with the bandwidth and priority needed to fulfill any QoS requirement. Furthermore, the FI can be used to safely set up point-to-point channels $([7,6])$, and to implement an efficient muli-hop broadcast mechanism, as described next.

\subsection{Multi-Hop Broadcast}

The ADHOC-MAC operation can be extended to implement multi-hop broadcast services over the whole network. The required relaying function, usually implemented at the network layer, can be effectively implemented at MAC layer using the connectivity information provided by the FI. Note that an effective multi-hop broadcast support at layer 2 can ease the design of routing protocols at the network layer.

As with flooding, the broadcast packets in the ADHOC-MAC network need to be numbered and the relaying procedure is applied only the first time the broadcast packet is received by a terminal. Let $\mathcal{C}_{i}$ be the set of neighbors of terminal $i$, i.e. all the terminals in the same $\mathrm{OH}$-cluster, and $\mathcal{C}_{j}^{i}$, for any $j \in \mathcal{C}_{i}$, the sets of neighbors' neighbors. Given that terminal $i$ receives a broadcast packet from terminal $z$ in slot $k$, we define the set of neighbors that have not received the packet in slot $k$ by $\mathcal{S}_{i} \subset \mathcal{C}_{j}^{i}$. All these sets are identified by terminal $i$ through the information carried by the FIs received in the $N$ slots following slot $k$.

At slot $k+N$, terminal $i$ recognizes whether or not it needs to relay the broadcast packets. Terminal $i$ does not relay the packet if $\mathcal{S}_{i}=\phi$ or if, for at least one $j \in\left(\mathcal{C}_{j}^{i}-\mathcal{S}_{i}\right)$ the following condition is satisfied:

$$
\mathcal{S}_{i} \subseteq \mathcal{C}_{j}^{i} \text { AND }\left(\left|\mathcal{C}_{j}^{i}\right|>\left|\mathcal{C}_{i}\right| \text { OR }\left(\left|\mathcal{C}_{j}^{i}\right|=\left|\mathcal{C}_{i}\right| \text { AND } I D_{j}>I D_{i}\right)\right) .
$$

where $I D_{i}$ denotes the address of terminal $i$. Basically, terminal $i$ does not relay the packet if set $\mathcal{S}_{i}$ is a subset of $\mathcal{C}_{j}^{i}$ and if either $\mathcal{C}_{j}^{i}$ has higher cardinality than $\mathcal{C}_{i}$ or, having the same cardinality, the address of $j$ is higher than the address of $i$.

\subsection{Topology Control in ADHOC MAC}

The need for an effective network topology control has been widely discussed in Section 3 and will be further supported by the numerical results presented in the next section.

Hereafter we present a topology control algorithm that can be easily implemented using the distributed information provided by the FI in ADHOC MAC.

The algorithm works in a fully distributed way by updating at each Power Control Period $(P C P)$ the transmitting power of each terminal in order to keep active a given number of bidirectional links between each terminal and its neighbors. Two terminals are connected by a 
bidirectional link if and only if they are within the transmitting range one another. The parameters of the algorithm are: $K$, the target number of bidirectional links, the value of the $P C P$ and the power ramping step $P_{\text {step }}$ which represents the minimum amount of power increase.

Using the information contained in the FI, each terminal updates a list $\alpha$ of neighboring nodes ordered according to the received power, from the higher to the lower. In order to reach this goal, each terminal should insert in its FI the information on its transmission power level.

At the end of each $P C P$ a terminal collects all the FIs received in the previous frame. If $N_{r}$ is the number of these received FIs, the terminal adjusts its transmitting power according to the following rules:

Rule 1: If $N_{r} \leq K$, the terminal increments its transmission power of $P_{\text {step }}$, and signals that $N_{r} \leq K$ setting the connection flag to $O$ in its own FI.

The transmitted power cannot exceed a maximum value $P_{\max }$. The connection flag in the FIs signals the connectivity status of the transmitter to its neighbors.

Rule 2: If $N_{r}=K$, the terminal sets its transmitting power so that to reach the $K$-th neighbor in its list. It signals $N_{r}=K$ by setting the connection flag to 1 in its $F I$.

Rule 3 : If $N_{r}>K$, for each of the last $N_{r}-K$ neighbors in list $\alpha$ it adds the neighbor to a subset $F$ if in the neighbor's FI the connection flag is set to 0 or is set to 1 and the ID of the terminal itself is signalled. If subset $F$ is empty the terminal sets its transmission power to reach the $K$-th neighbor of the ordered list and sets the connection flag in its FI to 1 , otherwise it sets its transmission power so to reach the most distant neighbor belonging to subset $F$ and sets the connection flag in its FI to 2.

The rationale of the algorithm is to maintain the number of bidirectional links equal to $K$ for each terminal. A terminal is allowed to have more than $K$ bidirectional links (Rule 3 ) only to keep the connectivity status of its neighbors above the quality constraint $\left(N_{r} \geq K\right)$.

In order to implement the proposed algorithm in ADHOC MAC the information carried by the FI should be enhanced. As already pointed out, each terminal should include in its FI its transmitting power level, which can be coded using 1 byte. Furthermore, a two bits connection flag must be added.

\section{Performance Evaluation}

In this section we present preliminary performance evaluations on the effectiveness of ADHOC MAC with respect to the single-hop broadcast service (Section 5.1) and multi-hop broadcast service (Section 5.2). Furthermore, we study the impact of the topology control algorithm presented in the previous section on the dynamics of ADHOC MAC (Section 5.3).

All the numerical results have been obtained by simulating the ADHOC MAC protocol in a network scenario where the terminals are dynamically generated according to a Poisson process and are placed in a square area with side length $A=1 \mathrm{Km}$ according to a uniformly distributed probability density. To avoid border effects, the square area is shaped like a wraparound surface.

Upon generation, each terminal tries to acquire its BCHs and, after $L=300$ frames have elapsed, it dies whether it has acquired the $\mathrm{BCH}$ or not. Each terminal has a transmission radius $R$ and each frame is composed by $N$ transmission slots. 


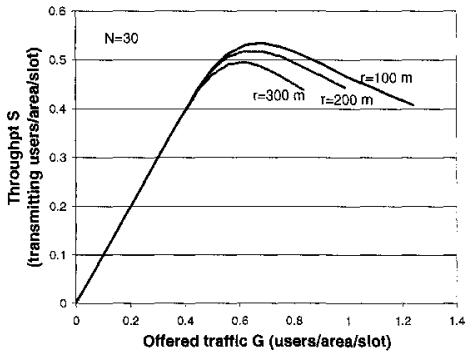

Figure 3: The throughput of $B C H$ channels versus the channel load for a torus surface, different coverage radii and frar

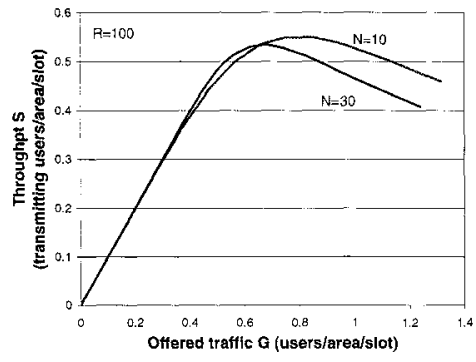

Figure 4: The throughput of $B C H$ channels versus the channel load for a torus surface, for different izes $N$.

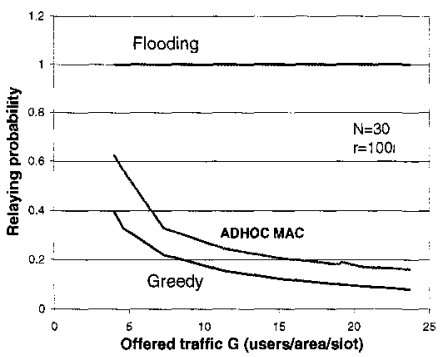

Figure 5: Packet relaying probability versus the average number of neighbors in a square region with edge $=1 \mathrm{Km}$ and with coverage radius $=100 \mathrm{~m}$.

\subsection{Single Hop Broadcast Efficiency}

According to ADHOC MAC, a terminal can support the single-hop broadcast service once it has acquired a $\mathrm{BCH}$ channel. To evaluate the efficiency of such a procedure we measure the throughput of $\mathrm{BCH}$ defined as the average number of terminals that use the channel per slot per unit area. In general, this figure is function of the offered traffic and depends on system parameters such as $N$ and $L$.

Figure 3 shows the throughput $S$ versus the offered traffic $G$ for $N=30$, and $R=$ $100,200,300 \mathrm{~m}$. The throughput reaches a maximum value $S_{\max }$ and shows an unstable behavior due to congestion caused by repeated access attempts.

We have observed that the $S_{\max }$ is much smaller than 1 because the protocol prevents the use of some slots to avoid hidden terminal collisions, thus limiting the radio resources reuse. This is the overhead one must pay to guarantee a reliable single hop broadcast. This overhead, as observed in Figure 3, changes with $R$. In fact, as $R$ decreases, the probability of slot reuse in disjoint $\mathrm{OH}-\mathrm{Clusters} \mathrm{increases,} \mathrm{but} \mathrm{reaches} \mathrm{a} \mathrm{saturation} \mathrm{value} \mathrm{for} A / R \geq 10$.

On the other hand, if $R$ is comparable with $\mathrm{A}, S_{\max }$ increases again because the number of terminals outside the OH-Cluster reduces. In the unrealistic case of $R=A, S_{\max }$ would be equal to 1 since all terminals are within a single $\mathrm{OH}$-Cluster.

Figure 4 shows $\mathbf{S}$ versus $\mathrm{G}$ for $N=10,30$. In general, a larger frame size should favor the throughput since it easier to find a free slot when a new channel is added at a given throughput $S$. However, a larger frame means proportionally larger users densit, which reduces reuse. 
In fact, adding users in the plane can transform non-overlapping $\mathrm{OH}$-clusters into overlapping ones and users that originally used the same slot must use different slots when merged into the TH-cluster. When $G<G_{\max }$ the former effect is predominant, whereas the second prevails in the congested zone. A further effect, though with less impact on throughput, is the reduction of the overhead due to collisions when a large frame length is adopted.

\subsection{Multi-Hop Broadcast efficiency}

As described in section 4.2, ADHOC-MAC provides a simple method to attain an effective multi-hop broadcast service, exploiting the distributed information transmitted in the FI fields. To quantitatively evaluate such an efficiency, we measured the number of retransmissions to ensure that a broadcast packet reaches all destinations. The numerical results obtained by simulation are presented in Figure 5 that shows the probability that a terminal retransmits a broadcast packet, i.e., the fraction of terminals involved in the broadcast relaying function, versus $G$, for $N=30$ and $R=100 \mathrm{~m}$.

For comparison purposes, we show the performance of the flooding technique and a centralized greedy approach. The former, adopted in IEEE 802.11, requires each terminal to retransmit a broadcast packet once and thus represent an upper bound for the fraction of terminals involved in the relaying function. The lower bound, more interesting for actual efficiency evaluation, is provided by the solution of the broadcast retransmissions minimization problem, which has been proven to be NP-hard [19]. Instead, we have used a suboptimal solution obtained by a centralized greedy procedure.

In the heuristic we used, the terminal with the higher number of neighbors still missing the broadcast packet is selected to perform the retransmission at each iteration step. The procedure terminates when all the terminals have received the packet. Such a procedure would require at each terminal the full knowledge of the network topology and a central controller should further coordinate the relaying phase.

The numerical results of Figure 5 have been obtained assuming a network topology composed by a regular square grid of nodes at a distance equal to terminal radio range, so that each node has four grid neighbors. The grid is introduced to guarantee the complete network connectivity. Additional nodes are generated randomly in order to change the offered traffic.

With both the greedy algorithm and the ADHOC MAC multi-hop algorithm the relaying probability decreases as the load increases. In fact, as $\mathrm{G}$ increases, the average number of neighbors increases and thus the fraction of terminals that need to retransmit decreases.

We note that $\mathrm{ADHOC}$ MAC performs closely to the greedy algorithm and, for a high degree of connectivity, ADHOC MAC provides a gain of $85 \%$ in terms of overhead reduction with respect to flooding.

\subsection{Topology Control Algorithm Efficiency}

The performance analysis of the topology control algorithm has been split into two traffic configurations: static and dynamic. In the static configuration the number of the terminals within the network is fixed throughout the simulation. In this scenario we validated the correctness of the topology control algorithm and we tested its convergence time. To do this, we randomly generated $M$ terminals within the network at the same time and we run ADHOC MAC with the topology control algorithm.

In the dynamic scenario the terminals enter and leave the network randomly. In this configuration we evaluated the impact of the topology control algorithm on the performance of ADHOC MAC when varying the traffic intensity and the parameter $K$.

The standard setting of the parameters used in the following simulations is: $L=300 \mathrm{frames}$ $N=30$ slots $/$ frame, Step $=1 \mathrm{~m}, P C P=5$ frames and $P_{\text {max }}$ is set to reach a distance of $250 \mathrm{~m}$ in square simulation area with edge equal to $1 \mathrm{Km}$. 


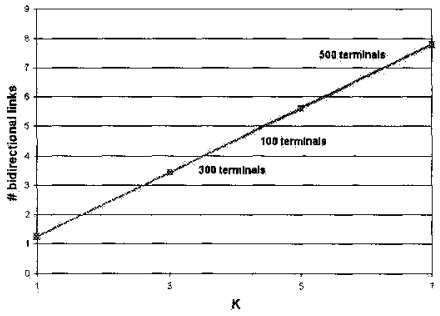

Figure 6: Number of bidirectional links versus $K$ for three cases of users' density in the standard setting.

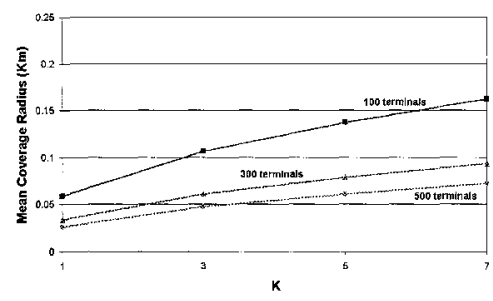

Figure 7: Average coverage radius versus $K$ for three cases of users' density in the standard setting.

\subsubsection{Static Analysis}

Figure 6 gives the average number of the actual installed bidirectional links versus $K$ for different values of terminals density $\left(\sigma=100,300,500\right.$ terminals $/ \mathrm{Km}^{2}$ ). As expected, the topology control algorithm adapts the transmitted power according to the terminals' density and provides in all the cases the same number of bidirectional links.

Figure 7 shows the average value of the terminals' coverage radius versus $K$. For a given $K$ the average value of the coverage radius grows as the terminals' density decreases. The required power to reach $K$ neighbors is inversely proportional to the network density. On the other hand, when fixing the network density, the value of the coverage radius increases with the parameter $K$, i.e., the average transmitting power grows if the number of required neighbors grows.

\subsubsection{Dynamic Analysis}

The throughput of the single-hop broadcast service provided by ADHOC MAC that has been measured with a fixed transmission radius shows an unstable behavior when the offered traffic increases, as observed in Figure 3. If the topology control algorithm behaves correctly, the throughput degradation as $\mathrm{G}$ increases should disappear. This behavior has been verified by the numerical results shown in Figure 8 which gives the network throughput $\mathrm{S}$ Net (number of transmissions per slot all over the network) versus the network offered traffic G Net for the two cases with and without the topology control algorithm. The throughput measured for $K=5,7,9$ is the same and linearly increases with the offered traffic.

The higher throughput with respect to the case of constant $R=100 \mathrm{~m}$ is mainly due to the the topology control algorithm that, by reducing the coverage radius as the offered traffic increases, favors the radio resource reuse and limits the access collisions.

These two phenomena have been monitored in our simulations.

Figure 9 shows the average coverage radius versus the G Net. The topology control shrinks the terminals' coverage radius when $G$ Net increases, with a consequent higher possibility of reusing the same slot all over the network.

The access collision probability versus $\mathrm{G}$ Net is shown in Figure 10. With the fixed coverage radius a sharp increase in the collisions is observed for high $\mathrm{G}$ Net values (corresponding to the unstable conditions of Figure 8). On the other hand, the topology control algorithm practically maintain the collision probability constant. 


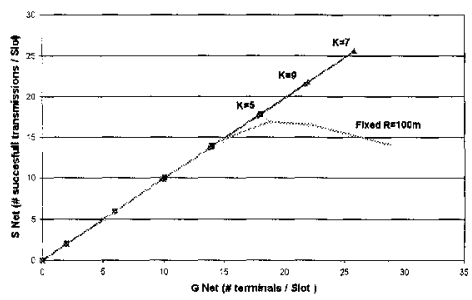

Figure 8: SNet versus $G$ Net for the cases with $(K=3,5,7)$ and without (coverage radius $R=100 \mathrm{~m}$ ) topology control in the standard setting.

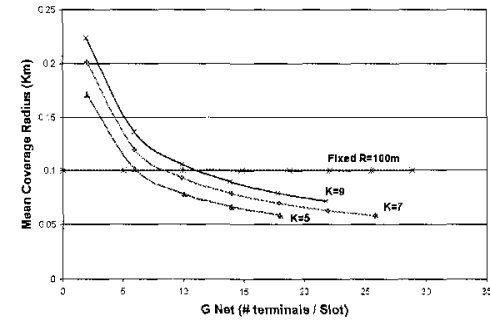

Figure 9: Average value of the coverage radius versus $G$ Net for the cases with $(K=3,5,7)$ and without (coverage radius $R=100 \mathrm{~m}$ ) topology control in the standard setting.

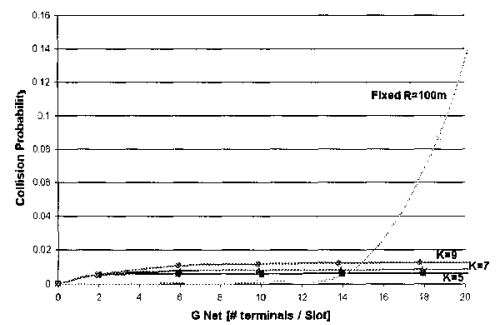

Figure 10: Percentage of collisions versus $G$ Net for the cases with $(K=3,5,7)$ and without (coverage radius $R=100 \mathrm{~m}$ ) topology control in the standard setting.

\section{Conclusions}

The provision of effective broadcast services within vehicles is a central point for designing applications like traffic control and accident signaling in IVC. The ADHOC MAC is one of the few MAC protocols able to provide a reliable single hop broadcast channel within mobile terminals, according to which any broadcast transmission is guaranteed to be correctly received by all the neighboring nodes of the transmitter. Furthermore, thanks to the connectivity information exchange within ADHOC MAC, effective algorithms for multi-hop broadcast transmissions and topology control purposes can be designed.

In this paper we have reviewed the capabilities of ADHOC MAC in providing broadcast services whose performances have been thoroughly analyzed through simulation. Further on, we have proposed a novel topology control algorithm able to dynamically adapt to network changes due to terminal mobility which can be easily integrated within ADHOC MAC framework. We have validated the algorithm by testing its behavior in static traffic environment and finally we have shown its good performance with dynamic traffic.

\section{References}

[1] "Special issue on intelligent vehicle highway systems", IEEE Transactions on Vehicular Technology, vol. 40, no. 1, 1991.

[2] European Transport Safety Council (ETSC), "Intelligent Transportation Systems and Road Safety Report", Brussels, Belgium, 1999.

[3] M. Aoki, "Inter-vehicle communication: technical issues on vehicle control applications", IEEE Communication Magazine, vol. 34, pp. 90-93, Oct. 1996. 
[4] I. Chisalita, N. Shahmehri, "A novel architecture for supporting vehicular communication", in Proc. of IEEE VTC 2002-Fall, vol. 2, pp. 1002-1006, Sept. 2002.

[5] R. Hager, R. Mathar, and J. Mattfeldt, "Intelligent cruise control and reliable communication of mobile stations", IEEE Transactions on Vehicular Technology, vol. 44, no. 3, pp. 443-448, 1995.

[6] F. Borgonovo, A. Capone, M. Cesana, L. Fratta, "ADHOC MAC: new MAC architecture for ad hoc networks providing efficient and reliable point-to-point and broadcast services", ACM Wireless Networks, to appear.

[7] F. Borgonovo, A. Capone, M. Cesana, L. Fratta, "ADHOC MAC: a new, flexible and reliable MAC Architecture for ad hoc Networks", In Proc. of IEEE WCNC, vol. 2, pp. 965-970, March 2003.

[8] I. Chlamtac and A. Farago, "Making Transmission Schedules Immune to Topology Changes in Multihop Packet Radio Networks", IEEE/ACM Transactions on Networking, vol. 2, no. 1, pp. 23-29, Feb. 1994.

[9] Chenxi Zhu, M. S. Corson, "A Five-Phase Reservation Protocol (FPRP) for Mobile Ad Hoc Networks", Wireless Networks, vol. 7, no. 4, September 2001.

[10] L. Roberts, "ALOHA Packet System With and Without Slots and Capture", Computer Communications Reviews, vol. 5, no. 2, pp. 28-42, April 1975.

[11] L. Kleinrock and F. Tobagi,"Packet Switching in Radio Channels. I. Carrier Sense Multiple Access Models and Their Throughput Delay Characteristics", IEEE Transanctions on Communications, vol. COM23, no. 12, pp. 1400-1416, Dec. 1975.

[12] IEEE 802.11 Working Group, "Part 11: Wireless LAN Medium Access Control (MAC) and Physical Layer (PHY) Specifications", ANSI/IEEE Std. 802.11, Sept. 1999.

[13] P. Karn, "MACA, A New Channel Access Protocol for Packet Radio", in Proc. of ARRL/CRRL Amateur Radio 9th Computer Networking Conference, pp. 134-140, 1990.

[14] V. Bharghavan et al., "MACAW: A Media Access Protocol forWireless LAN's", Computer Communications Reviews, vol. 24, no. 4, pp. 212-225, Oct. 1994.

[15] M. Impett, M.S. Corson, "A Receiver Oriented Approach to Reliable Broadcast in Ad Hoc Networks", in Proc. of IEEE WCNC 2000, Chicago, IL , USA, Sept. 2000

[16] S.T. Sheu, Y. Tsai, J. Chen, "A Highly Reliable Broadcast Scheme for IEEE 802.11 Multihop Ad Hoc Networks", in Proc. of IEEE ICC 2002, New York, NY, USA, April, 2002.

[17] K. Tang, M. Gerla, "Mac Layer Broadcast Support in 802.11 Wireless Networks", In Proc. of IEEE MILCOM 2000, 2000.

[18] I. Chlamtac, S. Kutten, "Tree-based Broadcasting in Multihop Radio Networks", IEEE Transactions on Communications, Oct 1987.

[19] Brad Williams, Tracy Camp, "Comparison of broadcasting techniques for mobile ad hoc networks", in Proc. of ACM MobiHOC, June 2002.

[20] Y. C. Tseng, S. Y. Ni , Y. S. Chen and J. P. Sheu, "The broadcast storm problem in a mobile ad hoc network", Wireless Networks, vol. 8, no. 2/3, March 2002.

[21] S. Agarwal, S. Krishnamurthy, R. H. Katz, S. K. Dao, "Distributed Power Control in Ad-hoc Wireless Networks, in Proc. of IEEE PIMRC 2001.

[22] E.S. Jung, N. H. Vaidya, A Power Control MAC Protocol for Ad Hoc Networks, in Proc. of ACM MOBICOM 2002, Atlanta, USA, September 2002.

[23] J.-P. Ebert, B. Stremmel, E. Wiederhold, A. Wolisz, An Energy-efficient Power Control Approach for WLANs, Journal of Communications and Networks (JCN), vol. 2, no. 3, pp. 197-206, Sept. 2000.

[24] J. P. Monks, V. Bharghavan, W. W. Hwu, A power controlled multiple access protocol for wireless packet networks, In Proc. of IEEE INFOCOM, April 2001

[25] S. Wu, Y. Tseng, J. Sheu, Intelligent Medium Access for mobile ad hoc networks with busy tones and power control, IEEE Journal on Selected Areas in Communications, vol. 18, no. 9 , pp. 1647-1657, Sept. 2000.

[26] R. Ramanathan, R. Rosales-Hain, Topology control of multihop wireless networks using transmit power adjustment, in Proc. of IEEE INFOCOM 2000, vol. 2, pp. 404-413, March

[27] M. Gerharz, C. deWaal, P. Martini, P. James, A cooperative nearest neighbours topology control algorithm for wireless Ad Hoc networks, in Proc. of ICCCN 2003, pp. 412-417,

[28] D. M. Blough, M. Leoncini, G. Resta, P. Santi, The k-neigh protocol for symmetric topology control in Ad Hoc networks, in Proc. of ACM MobiHoc 2003, pp. 141-152, June

[29] J. Liu, B. Li, Mobile Grid: capacity-aware topology control in mobile Ad Hoc networks, in Proc. of ICCCN 2002, pp. 570-574, Oct. 2002.

[30] R. Wattenhofer, L. Li, P. Bahl, Y. M. Wang, Distributed topology control for power efficient operation in multihop wireless Ad Hoc networks, in Proc. of IEEE INFOCOM 2001, vol.3, pp. 1388-1397, April 2001. 